\title{
Experts call for younger men to be offered PSA test for prostate cancer
}

In the fourth paragraph of this news story (BMJ 2016;352:i1802, doi:10.1136/bmj.i1802) we should have said that "Under current Public Health England rules, any man over 50 in England [not "the UK"] has the right to a PSA test if he requests it and considers the implications with his GP." 